\title{
MIXING IN BUBBLE COLUMN AND AIRLIFT REACTORS
}

\author{
A. SÁNCHEZ MIRÓN ${ }^{1}$, M.-C. CERÓN GARCÍA ${ }^{1}$, \\ F. GARCÍA CAMACHO ${ }^{1}$, E. MOLINA GRIMA ${ }^{\mathbf{1}}$ and Y. CHISTI ${ }^{2 *}$ \\ ${ }^{1}$ Department of Chemical Engineering, University of Almería, Almería, Spain \\ ${ }^{2}$ Institute of Technology and Engineering, Massey University, Palmerston North, New Zealand
}

$\mathrm{M}$

ixing behavior of three geometrically equivalent $0.06 \mathrm{~m}^{3}$ bubble column and airlift photobioreactors was compared for use in microalgal culture. Mixing was characterized in terms of the axial dispersion coefficient, mixing time, circulation time and Bodenstein number. Measurements were reported in tap water and sea water, as used in growing microalgal biomass. Compared with the two airlift reactors (a split-cylinder and a draft-tube sparged device, both of aspect ratio 9.1), the bubble column (aspect ratio $=9.1$ ) had a shorter mixing time at any aeration rate (superficial aeration velocity of up to $0.05 \mathrm{~m} \mathrm{~s}^{-1}$ in all reactors). Values of the various mixing parameters in the two fluids were quite comparable at identical aeration rates in any specific geometry of the reactors. Existing correlations of mixing times satisfactorily correlated the data in the bubble column, but not in the airlift reactors. The following correlations were established for the airlift devices:

$$
\begin{aligned}
& B o_{\mathrm{LG}}=\beta\left(F r^{1 / 3}\right)^{\lambda} \\
& B o=k\left(\frac{t_{\mathrm{m}}}{t_{\mathrm{c}}}\right)
\end{aligned}
$$

where $B o$ is the liquid-phase Bodenstein number, $\mathrm{Fr}$ is the Froude number, $B o_{\mathrm{LG}}$ is the Bodenstein number based on the superficial velocity of the gas, $t_{\mathrm{m}}$ is the mixing time, $t_{\mathrm{c}}$ is the circulation time, and $k, \beta$ and $\gamma$ are constants that depend on the geometry of the reactor and the fluid used.

Keywords: bubble columns; airlift bioreactors; mixing time; axial dispersion coefficient.

\section{INTRODUCTION}

Bubble columns and airlift devices have been advanced as effective photobioreactors for large-scale culture of microalgae (Sánchez Mirón et al., 1999, 2000). A knowledge of liquid-phase mixing times, liquid circulation velocities and axial mixing (characterized by axial dispersion coefficients and Bodenstein numbers) is important for design and operation of bubble column and airlift photobioreactors (Sánchez Mirón et al., 1999, 2000; Camacho Rubio et al., 2003). This work compares the mixing characteristics of three geometrically equivalent column photobioreactors for use in microalgal culture.

Mixing time, $t_{\mathrm{m}}$, is the time required to attain a given deviation from the fully mixed state from the instance of a tracer input. Mixing time is a global index of mixing

* Correspondence to: Professor Y. Chisti, Institute of Technology and Engineering PN456, Massey University, Private Bag 11 222, Palmerston North, New Zealand.

E-mail: Y.Chisti@massey.ac.nz and it is affected by axial and radial mixing and the effects of bulk flow. Mixing in airlift reactors is sometimes described in terms of a dimensionless mixing time $\theta_{\mathrm{m}}$, defined as follows:

$$
\theta_{\mathrm{m}}=\frac{t_{\mathrm{m}}}{t_{\mathrm{c}}}
$$

where $t_{\mathrm{c}}$ is the cycling time, or the time required for one passage through the circulation loop. The dimensionless mixing time is generally observed to depend only on the reactor geometry and not on gas velocity (Chisti, 1989).

Mixing in the axial direction in a bubble column is characterized by an axial dispersion coefficient, $E_{z}$, which is mainly a function of aeration rate, the geometry of the column and the properties of the fluid (Deckwer et al., 1974; Shah et al., 1982). In airlift bioreactors, the riser and downcomer zones can have different values of $E_{z}$ under given conditions of operation (Chisti, 1989). 
In addition, in an airlift loop, an overall value of $E_{z}$ can be identified for the entire circulation pathway (Blenke, 1979; Chisti, 1989). This overall $E_{z}$ is an averaged value which is related in some unknown manner to the $E_{z}$ values of the riser and the downcomer zones. An axial dispersion coefficient in a gas-liquid system characterizes the axial mixing produced by rising gas bubbles that carry elements of circulating fluid in bubble wakes. Because bubble rise faster than the liquid, a certain amount of liquid is carried forward faster than the bulk flow of the liquid. This produces mixing in the axial direction.

A Bodenstein number is the ratio between the mixing effects of the axial dispersion coefficient and the bulk movement of fluid. Bodenstein number $B o$ is defined as follows:

$$
B o=\frac{V_{\mathrm{L}} L}{E_{z}}
$$

where $V_{\mathrm{L}}$ is the linear velocity of the liquid, $L$ is the distance between tracer injection and detection points, and $E_{z}$ is the axial dispersion coefficient. A reactor is generally considered as perfectly mixed when $B o<0.1$. Plug flow occurs for $B o$ values of $>20$ (Chisti, 1989).

\section{MATERIALS AND METHODS}

\section{The Photobioreactors and Fluids}

The photobioreactors used included a bubble column, a split-cylinder airlift device and a concentric draft-tube airlift vessel sparged in the draft-tube (Figure 1). All vessels were made from $3.3 \mathrm{~mm}$ thick, transparent poly(methyl methacrylate), except for the lower $0.25 \mathrm{~m}$ sections that were made from stainless steel (Figure 1). The vessels were $0.193 \mathrm{~m}$ in internal diameter. The riser-to-downcomer cross sectional area ratio was unity for the split-cylinder and 1.24 for the draft-tube airlift vessel. The internal diameter of the draft-tube was $0.144 \mathrm{~m}$. The draft-tube and the baffle were located 0.091 and $0.096 \mathrm{~m}$ from the bottoms of the reactors, respectively. The dispersion height was $2 \mathrm{~m}$, for a working volume of $0.06 \mathrm{~m}^{3}$. The fluid was mixed by sparging with air through perforated pipe spargers (13-17 holes of $1 \mathrm{~mm}$ diameter; Figure 1). The superficial aeration velocity varied from 0.001 to about $0.05 \mathrm{~m} \mathrm{~s}^{-1}$, based on the total cross section of the reactors. The complete geometric details of the culture vessels appear in Figure 1 (Sánchez Mirón et al., 2000).

Measurements were made in tap water and the Mediterranean sea water. Studies in tap water allowed validation of some of the data by comparing with established correlations. Sea water was used because it is the medium
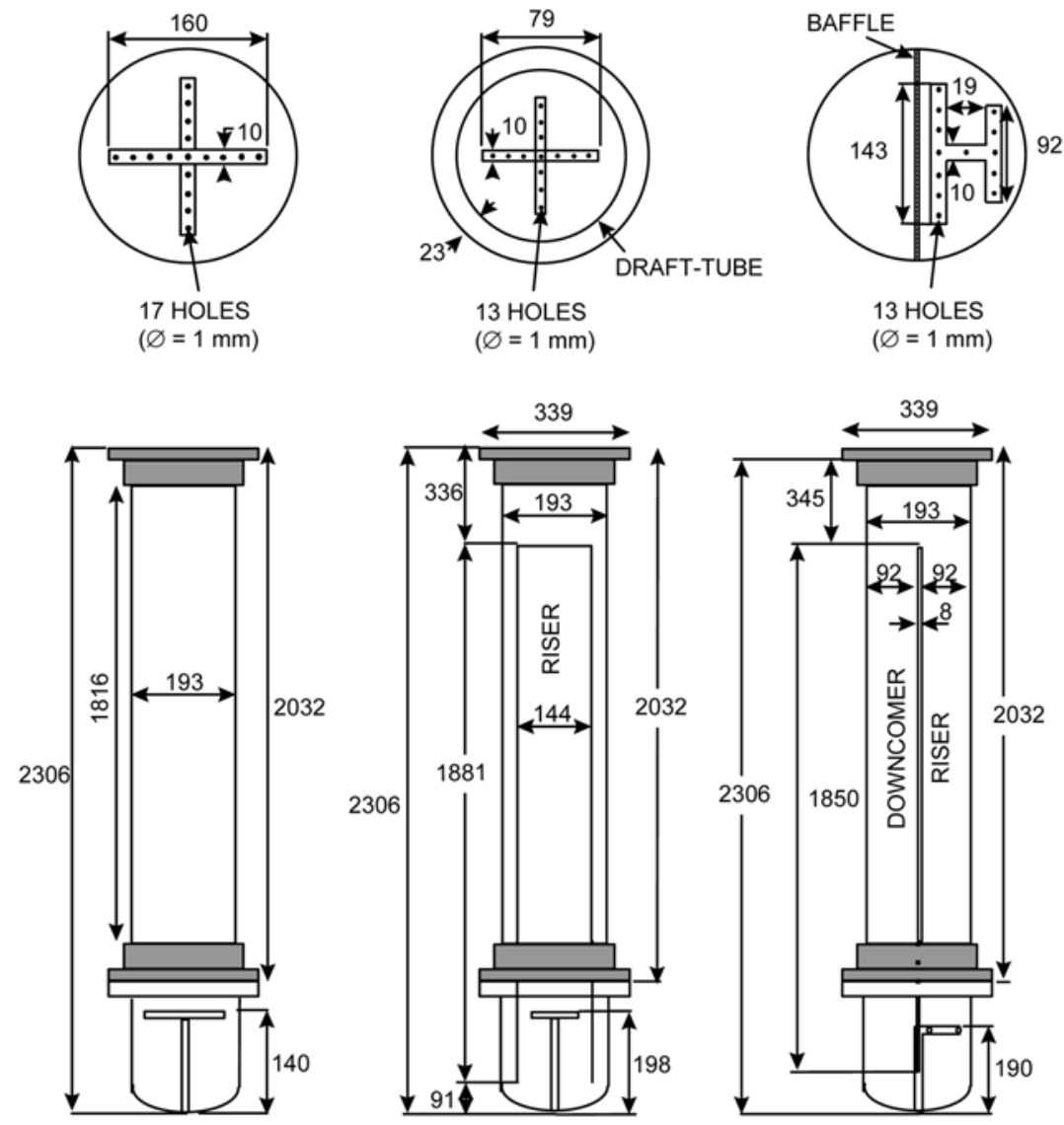

Bubble column

Draft-tube airlift

Split-cylinder

Figure 1. The geometry of photobioreactors and air spargers. All dimensions in mm. 
for culturing many microalgae (Sánchez Mirón et al., 1999). The composition of sea water has been published (Contreras et al., 1998). The viscosities of sea and tap water were $1.278 \times 10^{-3}$ and $0.998 \times 10^{-3} \mathrm{~Pa} \mathrm{~s}$, respectively. The viscosities were measured at $20^{\circ} \mathrm{C}$ using a Cannon-Fenske viscometer. The surface tension of the fluids was $72.5 \times 10^{-3}$ and $73.5 \times 10^{-3} \mathrm{~N} \mathrm{~m}^{-1}$ for sea and tap water, respectively. The surface tension was measured at $20^{\circ} \mathrm{C}$ using a platinum-iridium plate tensiometer (KÜSS, model K10DT, Germany).

\section{Liquid-phase Mixing Time}

The acid tracer method (Chisti, 1989) was used to measure the mixing time, defined as the time required to attain a 5\% deviation from complete homogeneity from the instance of tracer addition. After the reactor had been filled with the appropriate fluid, the $\mathrm{pH}$ was lowered to 2 by adding hydrochloric acid $(35 \% \mathrm{w} / \mathrm{v})$. The vessel was then bubbled with air $\left(U_{\mathrm{G}} \approx 0.03 \mathrm{~m} \mathrm{~s}^{-1}\right)$ for $20 \mathrm{~min}$ to remove any carbonates as carbon dioxide. The $\mathrm{pH}$ was now raised to $\mathrm{pH} 4.5$ by adding $12 \mathrm{M}$ sodium hydroxide. The acid tracer $(40 \mathrm{ml}$ of $35 \%$ hydrochloric acid) was now added instantaneously at the center of the surface of dispersion. The $\mathrm{pH}$ did not go below 2.0. The change in $\mathrm{pH}$ with time was measured using two $\mathrm{pH}$ electrodes located in the downcomer at 0.29 and $1.69 \mathrm{~m}$ below the surface of the gas-free liquid. The vertical distance between the electrodes was $1.40 \mathrm{~m}$. The $\mathrm{pH}$ data were recorded digitally using an IBM PC compatible computer. The dimensionless concentration, $C_{\mathrm{T}}$, of the tracer (hydrogen ion) was calculated as follows:

$$
C_{\mathrm{T}}=\left[\mathrm{H}^{+}\right]=\frac{\left[\mathrm{H}^{+}\right]_{\text {instantaneous }}-\left[\mathrm{H}^{+}\right]_{\text {initial }}}{\left[\mathrm{H}^{+}\right]_{\text {final }}-\left[\mathrm{H}^{+}\right]_{\text {initial }}}
$$

\section{Axial Dispersion Coefficient}

The axial dispersion coefficient, $E_{z}$, in the bubble column was calculated as described by Ohki and Inoue (1970), Towell and Ackerman (1972) and Deckwer et al. (1974). Thus, $E_{z}$ value was estimated as the best-fit value for reproducing the measured tracer-time profile according to the following equation:

$$
\begin{aligned}
\frac{C_{\mathrm{T}}}{C_{\mathrm{T} \text { final }}}= & 1+\frac{2 L}{\pi \delta} \sum_{n=1}^{\infty}\left[\frac{1}{n} \sin \left(\frac{n \pi \delta}{L}\right) \cos \left(\frac{n \pi z}{L}\right)\right. \\
& \left.\times \exp \left[-\left(\frac{n \pi}{L}\right)^{2} E_{z} t\right]\right]
\end{aligned}
$$

where $C_{\mathrm{T} \text { final }}$ is the final equilibrium dimensionless concentration of the tracer, $C_{\mathrm{T}}$ is the dimensionless tracer concentration [equation (3)] at time $t, z$ is the axial distance, $L$ is the distance from the surface of the fluid to the lower $\mathrm{pH}$ electrode, and $\delta$ is the increase in height of the static fluid caused by the addition of the tracer. In practice, six terms $(n=6)$ of equation (4) are sufficient for evaluating $E_{z}$ with an error of less than $1 \%$. Equation (4) is obtained by solving the one-dimensional diffusion model (Ohki and Inoue, 1970):

$$
\frac{\partial C_{\mathrm{T}}}{\partial t}=E_{z} \frac{\partial^{2} C_{\mathrm{T}}}{\partial z^{2}}
$$

For calculating the $E_{z}$ value in the riser-downcomer airlift loops, the overall value of $B o$ for the circulation loop was estimated using $B o$ as the fitting parameter to obtain a best match between the experimental acid tracer data and the following expression (Blenke, 1979):

$$
\sum_{p=1}^{\infty}\left(C_{\mathrm{T}}\right)_{p}=\left(\frac{B o}{4 \pi \theta}\right)^{1 / 2} \sum_{p=1}^{\infty} \exp \left[-B o(p-\theta)^{2} / 4 \theta\right]
$$

where $C_{\mathrm{T}}$ is the dimensionless tracer concentration [equation (3)], $\theta$ is dimensionless time [equation (7)] and $p$ is the dimensionless distance. At the instance of tracer input, $\theta=p=0$. The dimensionless time $\theta$ and the dimensionless distance $p$ are calculated as follows:

$$
\theta=\frac{t}{t_{\mathrm{c}}}
$$

and

$$
p=\frac{L_{\mathrm{t}}}{L_{\mathrm{c}}}
$$

In these equations, $t$ is the instantaneous time, $t_{\mathrm{c}}$ is the mean cycle time, $L_{\mathrm{t}}$ is the total distance travelled by the fluid at time $t$, and $L_{\mathrm{c}}$ is the length of the circulation loop. The overall $E_{z}$ value was now calculated using the $B o$ value, the measured liquid velocity $V_{\mathrm{L}}$ and the length of the circulation loop, as follows:

$$
E_{z}=\frac{V_{\mathrm{L}} L_{\mathrm{c}}}{B o}
$$

Equation (6) is obtained by solving a Fick's second law type diffusion model (Blenke, 1979):

$$
\frac{\partial C_{\mathrm{T}}}{\partial t}=E_{z} \frac{\partial^{2} C_{\mathrm{T}}}{\partial z^{2}}+V_{\mathrm{L}} \frac{\partial C_{\mathrm{T}}}{\partial z}
$$

A typical profile of dimensionless tracer concentration vs time is shown in Figure 2 for tap water in the split-cylinder airlift vessel at a superficial aeration velocity of $0.01 \mathrm{~m} \mathrm{~s}^{-1}$. Measured data and the fitted model profile [equation (6)] generally agreed closely, as shown in Figure 2.

\section{Liquid Circulation Velocity}

The average liquid circulation velocity $V_{\mathrm{L}}$ and the mean cycling time $t_{\mathrm{c}}$ in an airlift reactor are related as follows:

$$
V_{\mathrm{L}}=\frac{L_{\mathrm{c}}}{t_{\mathrm{c}}}
$$

where $L_{\mathrm{c}}$ is the length of the circulation loop. The value of $L_{\mathrm{c}}$ calculated from the geometry was approximately $3.5 \mathrm{~m}$ for both airlift vessels. The cycling time was estimated 


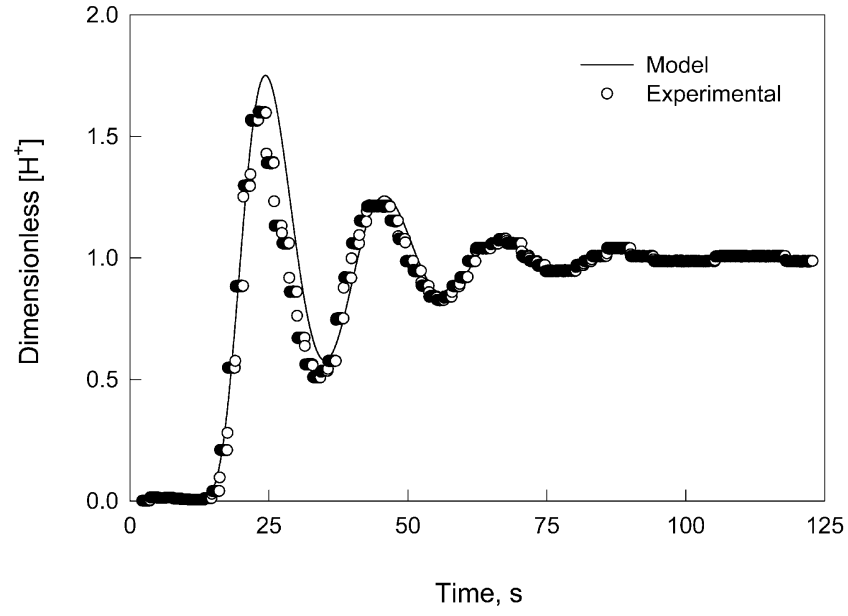

Figure 2. Measured and predicted tracer concentration profiles in the splitcylinder vessel (tap water, $U_{\mathrm{G}}=0.01 \mathrm{~m} \mathrm{~s}^{-1}$ ). Some symbols appear as solid circles because the data are close together and the edges of the symbols are superimposed.

as the time interval between adjacent peaks of a tracer concentration profile such as that shown in Figure 2. Signals from any one $\mathrm{pH}$ electrode were used to calculate $t_{\mathrm{c}}$. The linear liquid velocity in the downcomer $\left(V_{\mathrm{Ld}}\right)$ was calculated from the known vertical distance between the two $\mathrm{pH}$ electrodes and the time interval between the corresponding tracer peaks from the electrodes.

\section{RESULTS AND DISCUSSION}

\section{Mixing Time}

Mixing times vs aeration velocity data for the three reactors are shown in Figure 3. For a given reactor and aeration rate, the mixing time values in sea and tap water were quite similar (Figure 3), suggesting that changes in ionic strength above a certain minimal value did not significantly affect mixing behaviour. This concurs with similar observations about the effects of ionic strength on gas holdup (Chisti, 1989, 1998; Sánchez Mirón et al., 1999) that ultimately influences liquid circulation and mixing in pneumatically agitated gas-liquid reactors.

For a given aeration velocity, the bubble column always produced a shorter mixing time compared with the airlift reactors (Figure 3). Obviously, compared with the chaotic flow in the bubble column, the organized cyclic flow in the airlift reactors impeded bulk mixing. In all reactors, the general trend was a declining mixing time with increasing gas velocity or power input into the fluid. At incipient aeration, the mixing time in the airlift reactors was much more sensitive to aeration rate than the mixing time in the bubble column (Figure 3). The dependence of mixing time on aeration rate in the airlift reactors (Figure 3) was quite similar to the fluid cycling time profiles in the same reactors (Figure 4). A comparison of Figures 3 and 4 suggests that, in an airlift reactor, mixing improves if the circulation rate of the fluid is increased, i.e. $t_{\mathrm{c}}$ is reduced. This is because rapid cycling causes the fluid to pass more frequently through the relatively well-mixed head zone (Chisti, 1989) of the reactor (i.e. the zone above the
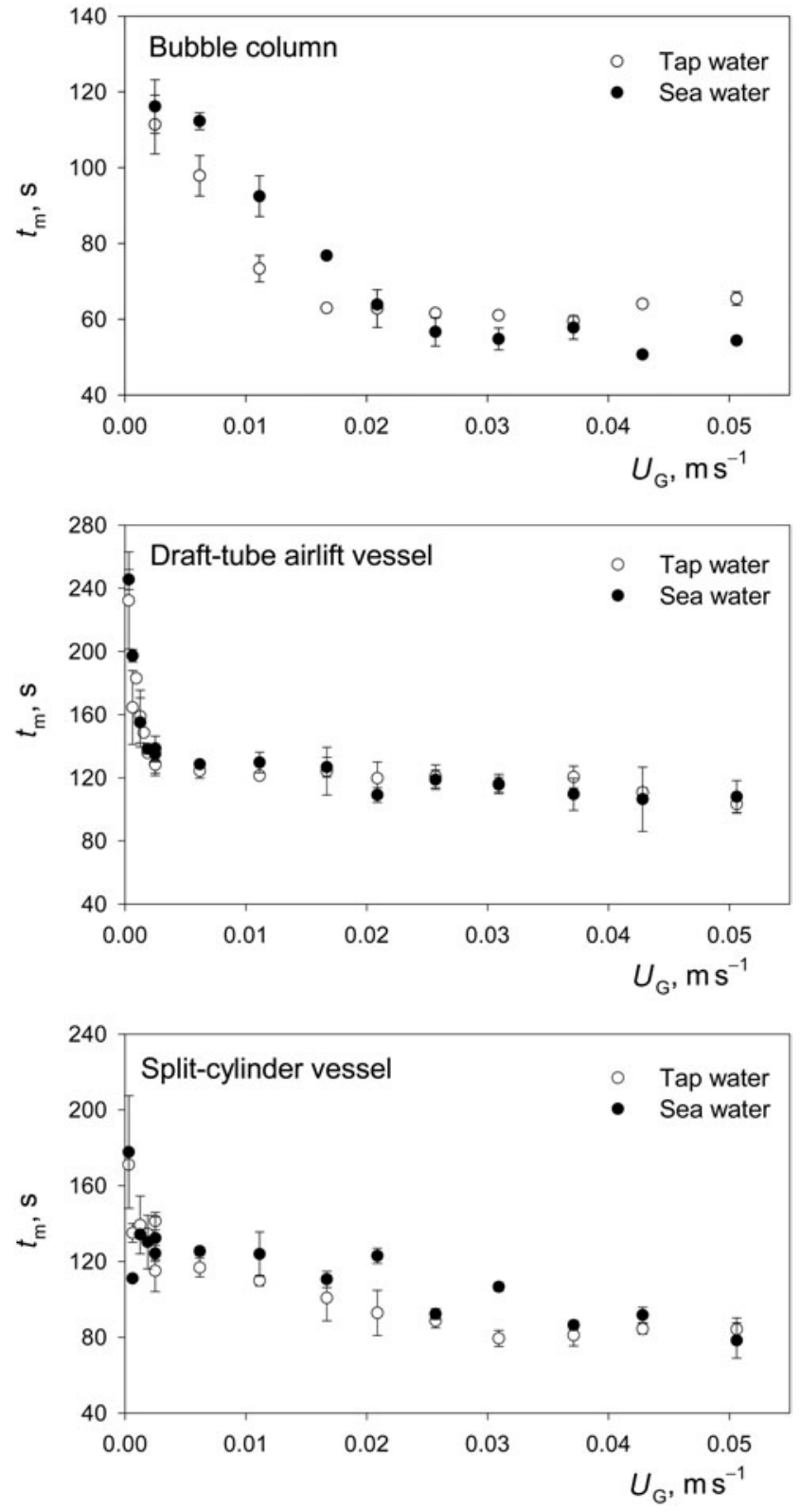

Figure 3. Mixing time variation with superficial aeration velocity.

upper edge of the draft-tube or baffle). Because the cycling time is sensitive to several geometric variables in an airlift reactor (Chisti et al., 1988; Chisti and Moo-Young, 1993), mixing time depends primarily on reactor geometry.

The slight increase in cycling time that is observed in Figure 4 with increasing $U_{\mathrm{Gr}}$ for $U_{\mathrm{Gr}}$ values of $\geq 0.02 \mathrm{~m} \mathrm{~s}^{-1}$ is quite typical of airlift reactors. This phenomenon is associated with micronization of gas bubbles because of increasing turbulence and a buildup of these fine bubbles in the downcomer zone. A consequently reduced difference between the gas holdup in the riser and downcomer reduces the driving force of liquid circulation (Chisti, 1989).

Although the effect of dispersion height above the baffle was not investigated in this work, this geometric variable is known to significantly influence mixing time (Chisti, 1989). Within limits, mixing time decreases with increasing dispersion height above the baffle (Chisti, 1989; Gavrilescu and Tudose, 1999). 

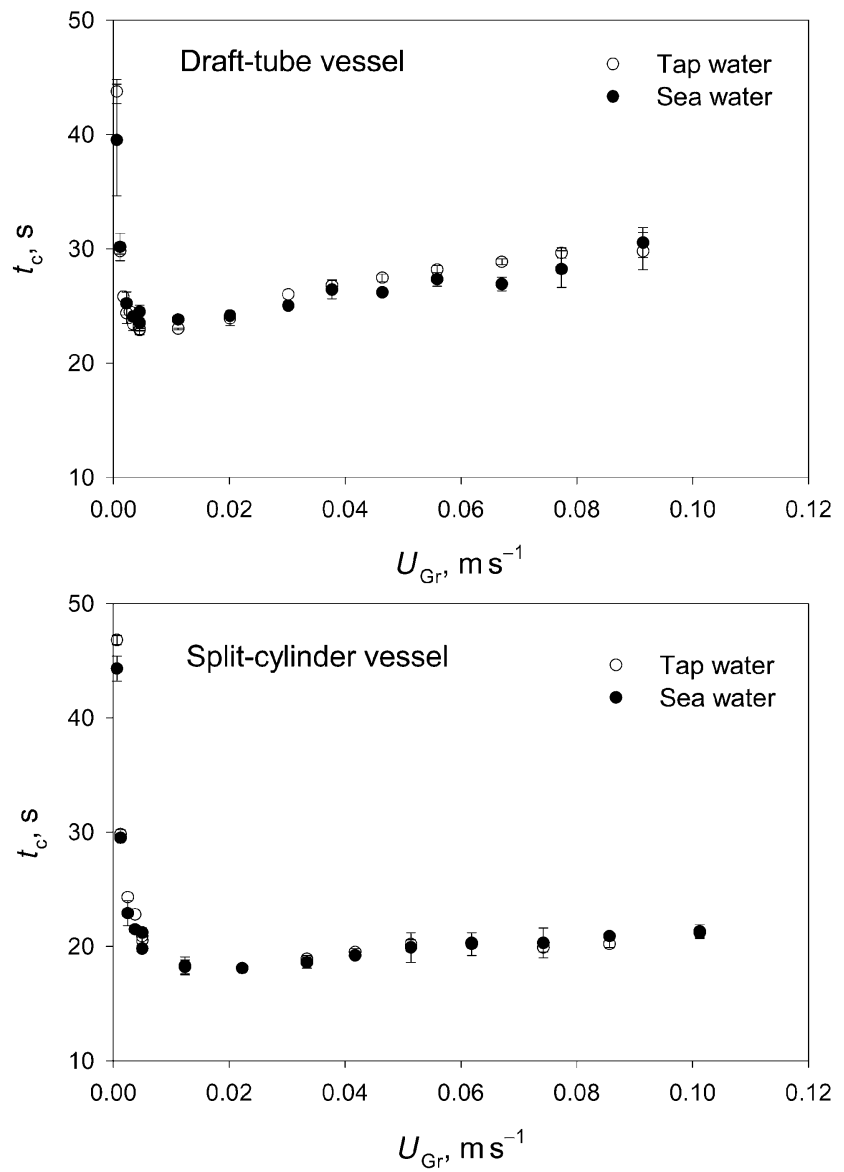

Figure 4. Cycling time variation with superficial gas velocity in the riser channel of the airlift reactors.

Reviews of mixing studies in airlift reactors (Chisti, 1989, 1998; Petrovic et al., 1995) document many correlations for predicting mixing times in these reactors; however, the available correlations have generally proved unsatisfactory for predicting anything other than the data used in obtaining them. For draft-tube airlift reactors, Bando et al. (1998) proposed the equation

$$
t_{\mathrm{m}}=c U_{\mathrm{G}}^{-0.5} d^{1.4}\left(\frac{h_{\mathrm{D}}}{d}\right)^{1.2}\left(\frac{d_{\mathrm{d}}}{d}\right)^{-1.4}\left(1-\frac{d_{\mathrm{d}}}{d}\right)^{-1.1}
$$

where the constant $c$ had a value of 2.2 (draft-tube sparged) or 2.6 (annulus sparged). In equation (12), $h_{\mathrm{D}}$ is the height of gas-liquid dispersion, $d$ is the diameter of the reactor vessel and $d_{\mathrm{d}}$ is the diameter of the draft-tube. Equation (12) applies when $0.11 \leq d \leq 0.50 \mathrm{~m}$ and $5 \leq h_{\mathrm{D}} / d \leq 40$. Figure 5 compares the predictions of equation (12) with the data measured in the draft-tube airlift reactor. Although equation (12) is consistent with the trend of the data (Figure 5), the equation overpredicts the data by a wide margin for the aeration velocity range $0<U_{\mathrm{G}}<0.01 \mathrm{~m} \mathrm{~s}^{-1}$, where the mixing time is most sensitive to aeration velocity.

\section{Axial Dispersion Coefficients}

A typical variation of the axial dispersion coefficient with aeration velocity is shown in Figure 6 for the bubble

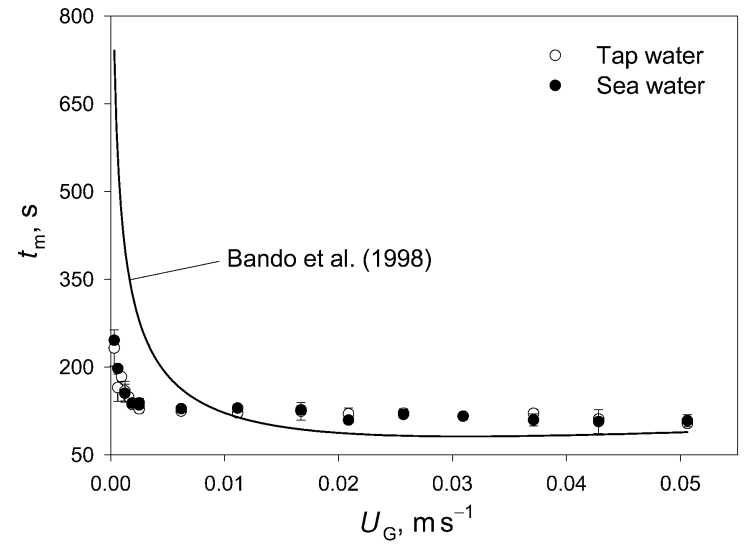

Figure 5. Comparison of the measured mixing times with predictions (solid line) of equation (12) in the draft-tube airlift reactor.

column and the draft-tube airlift vessel. In both cases, the $E_{z}$ values increased approximately hyperbolically with increasing aeration rate. In any one reactor, the $E_{z}$ values were similar for tap and sea water. Over almost the entire range of aeration rates used, the $E_{z}$ values in the bubble column were significantly greater than in the airlift device (Figure 6). This behaviour was consistent with the shorter mixing times in the bubble column than in the airlift reactors (Figure 3). Except at incipient aeration, the $E_{z}$
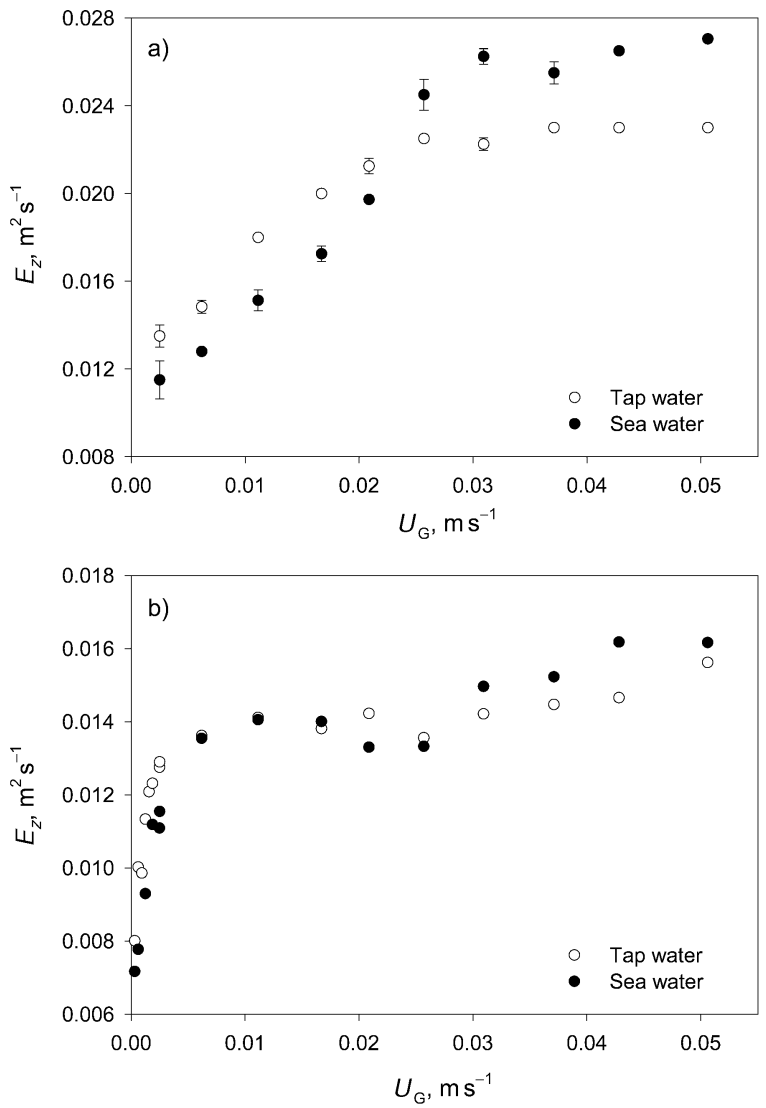

Figure 6. Axial dispersion coefficient vs aeration velocity: (a) bubble column; (b) draft-tube airlift vessel. 


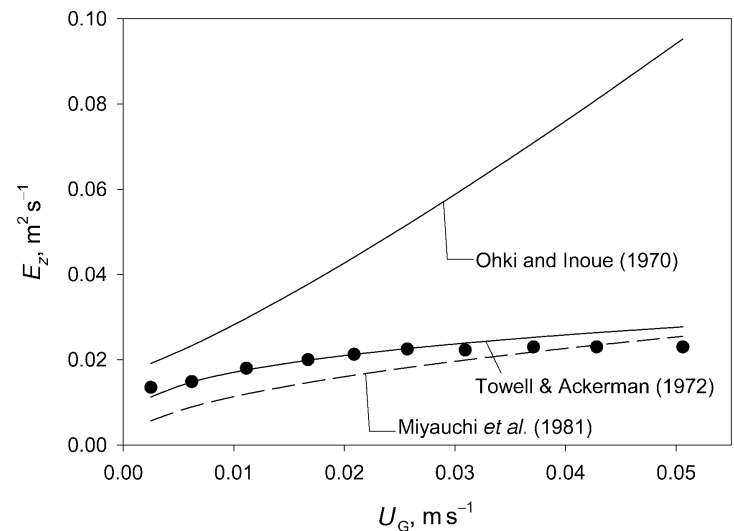

Figure 7. Comparison of measured bubble column $E_{z}$ values (tap water) with existing correlations.

values in the airlift reactor were relatively insensitive to changes in aeration velocity (Figure 6).

Figure 7 compares the $E_{z}$ values measured in the bubble column with predictions of the following correlations:

$$
\begin{aligned}
& E_{z}=0.3 d^{2} U_{\mathrm{G}}^{1.2}+170 d_{\mathrm{o}} \\
& E_{z}=2.7 d^{1.4} U_{\mathrm{G}}^{0.3} \\
& E_{z}=0.5 g^{1 / 4} U_{\mathrm{G}}^{1 / 2} d^{5 / 4}
\end{aligned}
$$

Equations (13)-(15) are due to Ohki and Inoue (1970), Towell and Ackerman (1972), and Miyauchi et al. (1981), respectively. The correlation of Towell and Ackerman
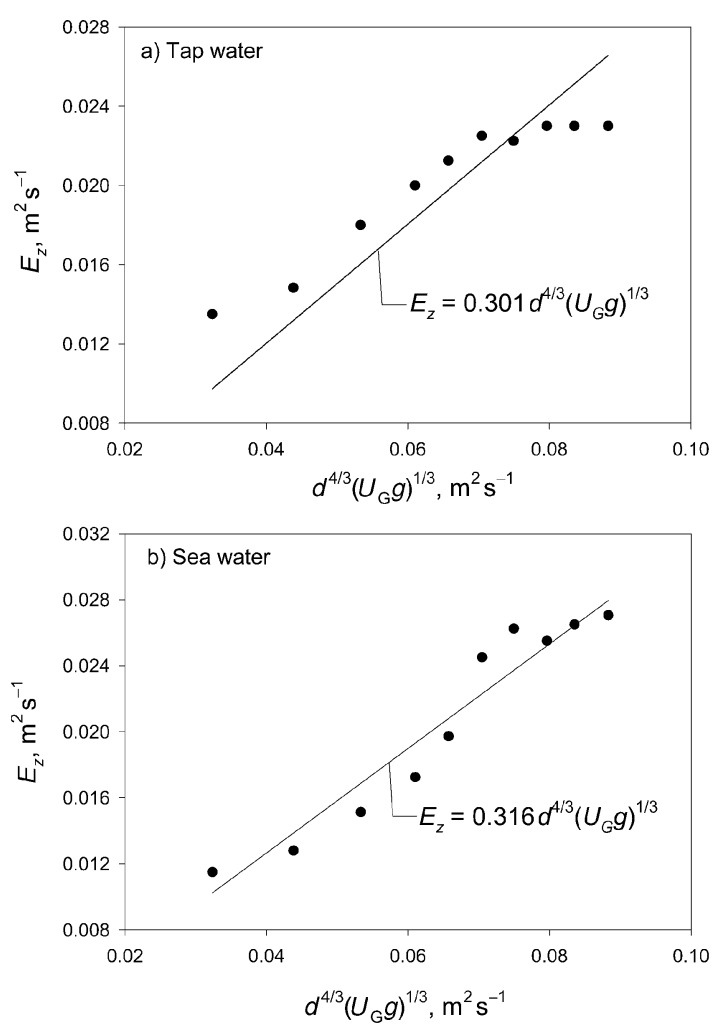

Figure 8. Comparison of measured bubble column $E_{z}$ values with equations of the form of equation (16): (a) tap water; (b) sea water. The regression coefficient values were 0.930 in both cases.
(1972) agreed quite closely with the measured data in both fluids. This is shown in Figure 7 for the data obtained in tap water. Compared with equation (14), equation (15) of Miyauchi et al. (1981) slightly underpredicted the data. Equation (13) was clearly unsatisfactory (Figure 7). In equations (13)-(15), $d_{\mathrm{o}}$ is the sparger hole diameter in $\mathrm{mm}$. The $U_{\mathrm{G}}, d$ and $g$ values are in $\mathrm{cm} \mathrm{s}^{-1}, \mathrm{~cm}$ and $\mathrm{cm} \mathrm{s}^{-2}$, respectively. The calculated $E_{z}$ value is in $\mathrm{cm}^{2} \mathrm{~s}^{-1}$.

Using an energy balance and the mixing length theory, Kawase and Moo-Young (1986) obtained the following equation for bubble columns:

$$
E_{z}=0.343 d^{4 / 3}\left(U_{\mathrm{G}} g\right)^{1 / 3}
$$

Similar equations satisfactorily correlated our data, as shown in Figure 8. Although equation (16) recommends a fixed exponent on the gas velocity term, empirical evidence (Deckwer et al., 1974) suggests this exponent to range from 0.3 to 1.2 , depending on the flow regime. Equation (16) is essentially the same as equation (14) although the latter equation is purely empirical. Equation (16) may slightly overestimate the $E_{z}$ values in bubble columns.

In comparison with equation (16), the data correlated (Figure 9) better with equations of the form

$$
B o_{\mathrm{LG}}=\beta\left(F r^{1 / 3}\right)^{\lambda}
$$

as recommended by Rice et al. (1981). In equation (17), $\beta$ and $\lambda$ are constants, $F r$ is the Froude number and Bodenstein
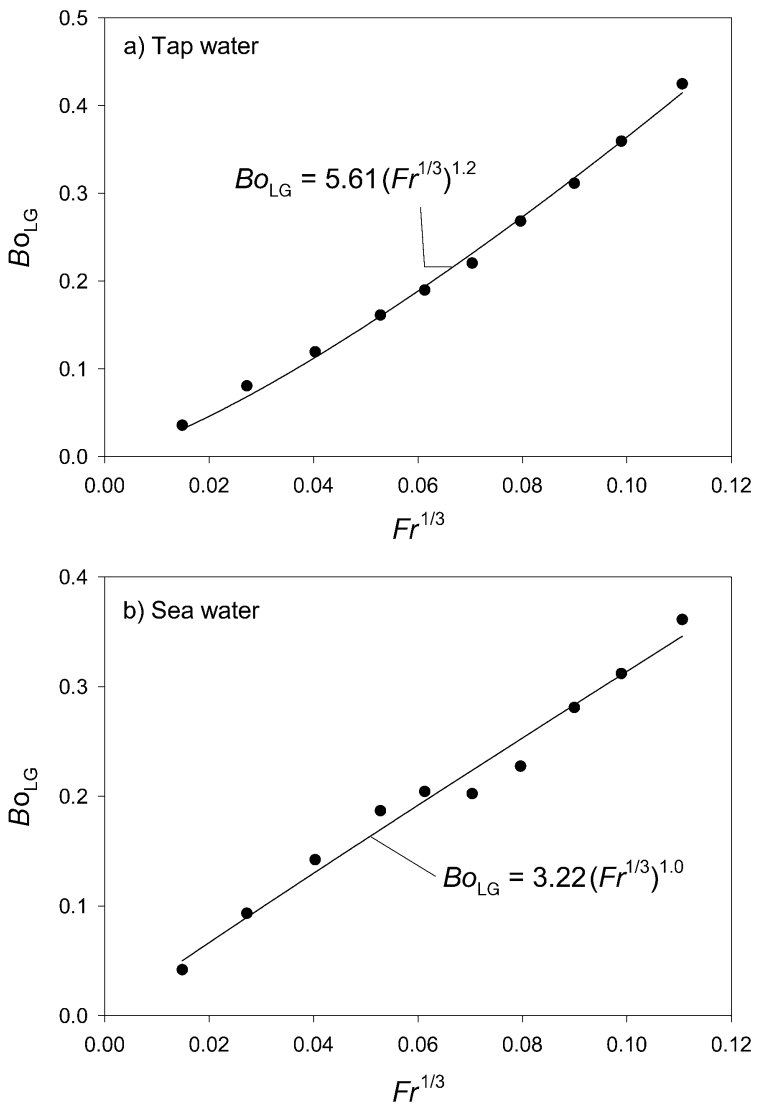

Figure 9. Improved fit of the bubble column $E_{z}$ values with equations of the form of equation (17): (a) tap water; (b) sea water. The regression coefficient values were 0.996 and 0.978 for (a) and (b), respectively. 
number is based on the superficial velocity of the gas. $\mathrm{Fr}$ and $B o_{\mathrm{LG}}$ are calculated as follows:

$$
\begin{aligned}
& F r=\frac{U_{\mathrm{G}}^{2}}{g d} \\
& B o_{\mathrm{LG}}=\frac{U_{\mathrm{G}} d}{E_{z}}
\end{aligned}
$$

Equation (17) uses $\beta$ and $\lambda$ as fitting parameters; thus, $E_{z} \propto\left(U_{\mathrm{G}}^{a} d^{b}\right)$ where $a$ and $b$ are interdependent. Equations (14) and (16) do not reveal this.
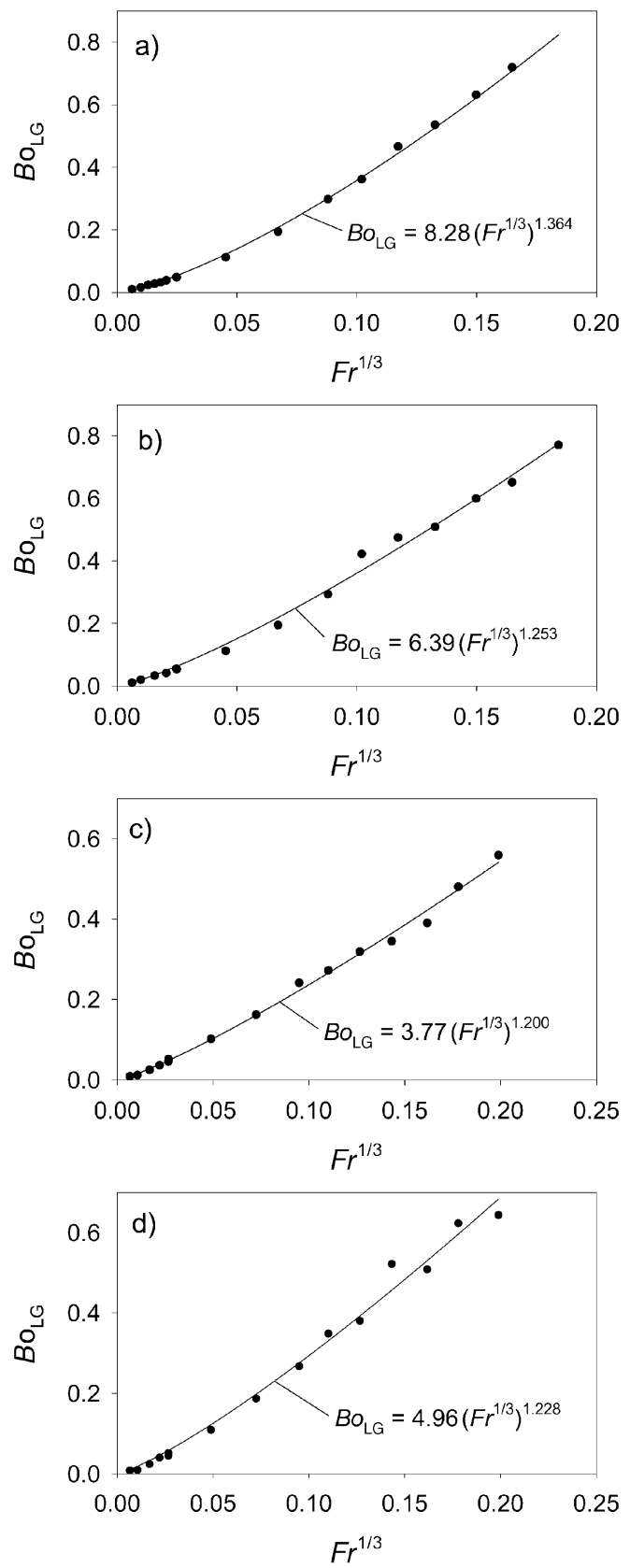

Figure 10. Correlation of $B o_{\mathrm{LG}}$ with $\mathrm{Fr}^{1 / 3}$ in airlift reactors: (a) draft-tube vessel with tap water; (b) draft-tube vessel with sea water; (c) splitcylinder with tap water; (d) split-cylinder with sea water. The regression coefficient values were (a) 0.999, (b) 0.994, (c) 0.995, and (d) 0.989 .
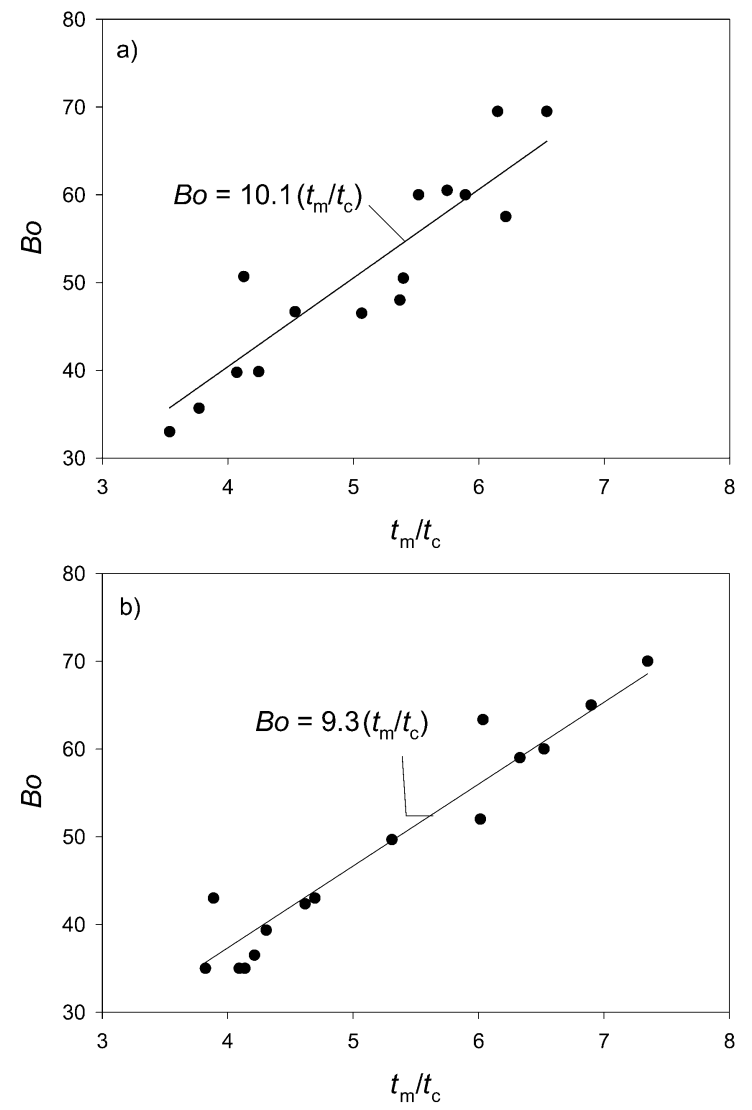

Figure 11. Correlation of Bo with $t_{\mathrm{m}} / t_{\mathrm{c}}$ in airlift reactors: (a) draft-tube vessel with sea water; (b) split-cylinder reactor with tap water. The regression coefficient values were 0.840 and 0.931 for (a) and (b), respectively.

As shown in Figure 10, equations of the same general form as equation (17) correlated exceptionally well the $B o_{\mathrm{LG}}$ values in both airlift reactors and fluids. In airlift reactors, the liquid phase Bodenstein number [equation (2)] correlated with the ratio of the mixing and cycling times, as shown in Figure 11 for both reactors and fluids. The form of the correlation is

$$
B o=k\left(\frac{t_{\mathrm{m}}}{t_{\mathrm{c}}}\right)
$$

where the mean $k$ value for both the airlift reactors and fluids was $9.6 \pm 0.2$. Because from the defining equations [equations (11) and (9)] the cycling time is

$$
t_{\mathrm{c}}=\frac{L_{\mathrm{c}}}{V_{\mathrm{L}}}
$$

and $B o$ is

$$
B o=\frac{V_{\mathrm{L}} L_{\mathrm{c}}}{E_{z}}
$$

equation (20) can be rewritten as follows:

$$
E_{z}=\gamma \frac{L_{\mathrm{c}}^{2}}{t_{\mathrm{m}}}
$$


where $\gamma$ is a constant. Thus, $E_{z}$ and mixing time are inversely related. This concurs with similar suggestions for bubble columns (Field and Davidson, 1980; Kawase and Moo-Young, 1989).

\section{CONCLUDING REMARKS}

In comparing mixing behaviour of relatively large geometrically equivalent bubble column and internal-loop airlift reactors, the bubble column was found to have shorter mixing times over the entire range of the aeration rates tested and in both fluids. The measured values of the liquid-phase axial dispersion coefficient in the bubble column agreed well with the correlations of Towell and Ackerman (1972) and Miyauchi et al. (1981), but not with the equation recommended by Ohki and Inoue (1970). The available correlations for mixing time in airlift reactors could not correlate the data closely. Correlations of the general form

$$
B o_{\mathrm{LG}}=\beta\left(F r^{1 / 3}\right)^{\lambda}
$$

and

$$
B o=k\left(\frac{t_{\mathrm{m}}}{t_{\mathrm{c}}}\right)
$$

proved exceptionally good in correlating the overall liquidphase dispersion coefficients for the riser-downcomer loop of the airlift reactors.

\section{NOMENCLATURE}

Bo

$B o_{\mathrm{LG}}$

$C_{\mathrm{T}}$

$C_{\mathrm{T} \text { final }}$

$c$

$d$

$d_{\mathrm{d}}$

$d_{\mathrm{o}}$

$E_{z}$

$\mathrm{Fr}$

$\left[\mathrm{H}^{+}\right]_{\text {fin }}$

$\left[\mathrm{H}^{+}\right]_{\text {initial }}$

$\left[\mathrm{H}^{+}\right]_{\text {instantaneous }}$

$h_{\mathrm{D}}$

$L_{\mathrm{c}}$

$L_{\mathrm{t}}$

$p$

$t_{\mathrm{m}}$

$U_{\mathrm{G}}$

$U_{\mathrm{G}}$

$V_{\mathrm{L}}$ liquid phase Bodenstein number defined by equation (2) Bodenstein number based on gas velocity and defined by equation (19)

dimensionless tracer concentration defined by equation (3)

final equilibrium dimensionless concentration of the tracer

constant in equation (12)

diameter of the reactor vessel, $\mathrm{m}$

diameter of the draft-tube, $m$

diameter of sparger holes, $\mathrm{mm}$

axial dispersion coefficient, $\mathrm{m}^{2} \mathrm{~s}^{-1}$

Froude number defined by equation (18)

gravitational acceleration, $\mathrm{m} \mathrm{s}^{-2}$

dimensionless tracer concentration defined by equation (3)

final tracer concentration, $\mathrm{kmol} \mathrm{m}^{-3}$

initial tracer concentration, $\mathrm{kmol} \mathrm{m}^{-3}$

instantaneous tracer concentration, $\mathrm{kmol} \mathrm{m}^{-3}$

height of gas-liquid dispersion, $\mathrm{m}$

constant in equation (20)

distance between tracer injection and detection points, $\mathrm{m}$

length of the circulation loop, $m$

total distance travelled by the fluid at time $t, \mathrm{~m}$

dimensionless distance defined by equation (8)

time or instantaneous time, $\mathrm{s}$

mean cycling time, $s$

mixing time, $\mathrm{s}$

superficial gas velocity based on the diameter of the reactor, $\mathrm{m} \mathrm{s}^{-1}$

superficial gas velocity based on the riser channel cross section, $\mathrm{m} \mathrm{s}^{-1}$

riser-downcomer averaged linear velocity of the liquid, $\mathrm{m} \mathrm{s}^{-1}$

linear liquid velocity in the downcomer, $\mathrm{m} \mathrm{s}^{-1}$

axial distance, $\mathrm{m}$

Greek symbols
$\beta$
$\gamma$
$\delta$
$\theta$
$\theta_{m}$
$\lambda$

parameter in equation (17)

constant in equation (23)

increase in height of the static fluid caused by the addition of the tracer, $m$

dimensionless time defined by equation (7)

dimensionless mixing time defined by equation (1)

parameter in equation (17)

\section{ACKNOWLEDGEMENTS}

This research was supported by the Comision Interministerial de Ciencia y Tecnología (CICYT) (BIO98-0522), Spain, and the European Union (Project BRPR CT97-0537).

\section{REFERENCES}

Bando, Y., Hayakawa, H. and Nishimura, M., 1995, Effects of equipment dimensions on liquid mixing time of bubble column with draft tube, J Chem Eng Jpn, 28: 225-227.

Blenke, H., 1979, Loop reactors, Adv Biochem Eng, 13: 121-214.

Camacho Rubio, F., García Camacho, F., Fernández Sevilla, J.M., Chisti, Y. and Molina Grima, E., 2003, A mechanistic model of photosynthesis in microalgae, Biotechnol Bioeng, 81: 459-473

Chisti, Y., 1989, Airlift Bioreactors (Elsevier, New York), p 355.

Chisti, Y., 1998, Pneumatically agitated bioreactors in industrial and environmental bioprocessing: hydrodynamics, hydraulics and transport phenomena, Appl Mech Rev, 51: 33-112.

Chisti, Y. and Moo-Young, M., 1993, Improve the performance of airlift reactors, Chem Eng Prog, 89(6): 38-45.

Chisti, Y., Halard, B. and Moo-Young, M., 1988, Liquid circulation in airlift reactors, Chem Eng Sci, 43: 451-457.

Contreras, A., García, F., Molina, E. and Merchuk, J.C., 1998, Interaction between $\mathrm{CO}_{2}$-mass transfer, light availability and hydrodynamic stress in the growth of Phaeodactylum tricornutum in a concentric tube airlift photobioreactor, Biotechnol Bioeng, 60: 317-325.

Deckwer, W.-D., Burckhart, R. and Zoll, G., 1974, Mixing and mass transfer in tall bubble columns, Chem Eng Sci, 29: 2177-2188.

Field, R.W. and Davidson, J.F., 1980, Axial dispersion in bubble columns, Trans IChemE, 58: 228-236.

Gavrilescu, M. and Tudose, R.Z., 1999, Modeling mixing parameters in concentric-tube airlift bioreactors. Part I: mixing time, Bioproc Eng, 20: $423-428$.

Kawase, Y. and Moo-Young, M., 1986, Influence of non-Newtonian flow behavior on mass transfer in bubble columns with and without draft tubes, Chem Eng Commun, 40: 67-83.

Kawase, Y. and Moo-Young, M., 1989, Mixing time in bioreactors, J Chem Technol Biotechnol, 44: 63-75.

Miyauchi, T., Furusaki, S., Morooka, S. and Ikeda, Y., 1981, Transport phenomena and reaction in fluidized catalyst beds, Adv Chem Eng, 11: $275-448$.

Ohki, Y. and Inoue, H., 1970, Longitudinal mixing of the liquid phase in bubble columns, Chem Eng Sci, 25: 1-16.

Petrovic, D.L., Pošarac, D. and Dudukovic, A., 1995, Prediction of mixing time in airlift reactors, Chem Eng Commun, 133: 1-9.

Rice, R.G., Tupperainen, J.M.I. and Hedge, R.M., 1981, Dispersion and holdup in bubble columns. Comparison of rigid and flexible spargers, Can J Chem Eng, 59: 677-687.

Sánchez Mirón, A., Contreras Gómez, A., García Camacho, F., Molina Grima, E. and Chisti, Y., 1999, Comparative evaluation of compact photobioreactors for large-scale monoculture of microalgae, J Biotechnol, 70: 249-270.

Sánchez Mirón, A., García Camacho, F., Contreras Gómez, A., Molina Grima, E. and Chisti, Y., 2000, Bubble column and airlift photobioreactors for algal culture, AIChE J, 46: 1872-1887.

Shah, Y.T., Kelkar, B.G., Godbole, S.P. and Deckwer, W.-D., 1982, Design parameters estimations for bubble column reactors, AIChE J, 28: 353-379.

Towell, G.D. and Ackerman, G.H., 1972, Axial mixing of liquid and gas in large bubble reactor, in Proceedings of 5th European/2nd International Symposium Chemical Reaction Engineering, p B3-1.

The manuscript was received 23 January 2004 and accepted for publication after revision 25 May 2004. 